\title{
Anti-inflammatory Effect of Flurbiprofen Tape Applied Percutaneously to Rats with Adjuvant-Induced Arthritis
}

\author{
Shinji Uchida, Takashi Morishita, Yutaka Ikeda and Toshihiro Akashi \\ Research Laboratory, Yutoku Pharmaceutical Industries Co., Ltd., Kashima, Saga 849-13, Japan \\ Received February 18, 1995 Accepted June 14, 1995
}

\begin{abstract}
The anti-inflammatory effect of flurbiprofen tape (FP-T) by topical application was investigated, and the findings were compared with the results of oral administration of flurbiprofen to adjuvant arthritic rats. The topical application of FP-T significantly suppressed both applied and non-applied hind paw edema, with a potency similar to that seen with the oral administration of flurbiprofen. Body weight also increased with these treatments. Plasma levels of flurbiprofen differed little between topical application of FP-T and oral administration of flurbiprofen. Gastric damage induced by topical application of FP-T was significantly less than that seen in case of oral administration of flurbiprofen. These results suggest that the anti-inflammatory effects of FP-T cannot be entirely explained by flurbiprofen permeating inflamed tissue below the application site; rather, flurbiprofen penetrating into the systemic circulation may explain these actions.
\end{abstract}

Keywords: Anti-inflammatory, Tape, Flurbiprofen, Transdermal penetration

A poultice or ointment containing non-steroidal antiinflammatory drugs (NSAIDs), such as indomethacin, ketoprofen and flurbiprofen, is widely used to treat patients with various local inflammatory diseases and arthropathies. These external drugs are characteristic in that their topical application has anti-inflammatory and analgesic effects on local inflamed tissue and also reduces adverse effects, such as gastric damage, because of the low blood concentration of the drugs $(1,2)$. The flurbiprofen tape (FP-T) is an adhesive tape containing $0.15 \mathrm{mg}$ of flurbiprofen per $\mathrm{cm}^{2}$, and it has a higher transdermal permeation capacity than other external materials. The antiinflammatory effects and plasma flurbiprofen concentrations after topical application of FP-T were compared with those after oral administration of flurbiprofen to rats with adjuvant arthritis. We also examined the effect of FP-T and flurbiprofen on rat gastric mucosae.

\section{MATERIALS AND METHODS}

\section{Materials}

Flurbiprofen and Mycobacterium butyricum were purchased from Kaken Pharma (Tokyo) and Difco Laboratories (Detroit, MI, USA), respectively.

\section{Preparation of FP-T}

Preparation of FP-T was as follows: Fifty-eight grams of natural rubber was dissolved in toluene, shaken at room temperature for $4 \mathrm{hr}$ and then stored overnight. Ten grams of polybutene, $14.7 \mathrm{~g}$ of liquid paraffin, $15 \mathrm{~g}$ of petroleum resin, $1.5 \mathrm{~g}$ of flurbiprofen and $0.8 \mathrm{~g}$ of peppermint oil were added to the solution, and the preparation was shaken for $3 \mathrm{hr}$ at room temperature. The mixture was coated onto polyethylene terephthalate film and dried for $3 \mathrm{hr}$ at room temperature. Nonwoven fabric was used for the backing film of FP-T. The content of flurbiprofen was $0.15 \mathrm{mg}$ per $\mathrm{cm}^{2}$, and the thickness was 100 $\mu \mathrm{m}$. Preliminarily, we examined the effects of FP-T containing several amounts of flurbiprofen on carrageenininduced paw edema in rats. The FP-T applied to the hind paw suppressed paw edema in a dose-dependent manner. From the findings, we decided that the content of flurbiprofen should be $0.15 \mathrm{mg}$ per $\mathrm{cm}^{2}$.

\section{Rat adjuvant arthritis}

Male Lewis rats, aged 6 weeks and purchased from Seiwa Experimental Animals (Fukuoka) were kept in an air-conditioned room $\left(22 \pm 11^{\circ} \mathrm{C}, 55 \pm 10 \%\right)$, with lights on $12 \mathrm{hr}$ per day (8:00-20:00). Standard chow and water were provided ad libtum for one week before the start of the experiments. Adjuvant arthritis was induced by the 
method of Winder et al. (3). In brief, rats were injected s.c. with $0.1 \mathrm{ml}$ of $0.6 \%$ Mycobacterium butyricum-liquid paraffin into the root of the tail. On day 20, the rats were selected according to the degree of swelling in the right and left hind paws and randomly assigned to three groups: Group $1(\mathrm{n}=10)$, non-treated control; Group 2 $(\mathrm{n}=10)$, topical application of FP-T $(3.5 \mathrm{~cm} \times 4 \mathrm{~cm})$; Group $3(n=10)$, oral administration of flurbiprofen $(0.75 \mathrm{mg} / \mathrm{kg})$. The FP-T was applied to the right hind paw for $6 \mathrm{hr}$ per day (10:00-16:00), and the flurbiprofen suspended in $5 \%$ gum arabic was orally administered once a day (10:00) on day 20 until day 28. After the FP-T was applied for $6 \mathrm{hr}$, the tapes were removed. The flurbiprofen content of the tapes removed was measured by using an HPLC system. The amount of flurbiprofen released was calculated from the flurbiprofen content of the tapes removed. The dose of FP-T calculated based on the release amount of flurbiprofen from FP-T was about 1 $\mathrm{mg} / \mathrm{kg} / \mathrm{day}$. The volumes of the right and left hind paw were measured using a plethysmometer (TK-102; Unicom, Chiba) on days $0,20,23$ and 27. The percentage of swelling was expressed as the ratio of swelling hind paw volume to the initial volume.

\section{Plasma concentration of furbiprofen}

On day 27, after the measurement of hind paw volume, drugs were again administered to the rats. Under ether anesthesia, $0.5 \mathrm{ml}$ of blood was withdrawn from an or- bital vein into a heparinized tube $1,3,6,8,12$ and $24 \mathrm{hr}$ after drug administration. The blood was centrifuged at $3,000 \mathrm{rpm}$ for $5 \mathrm{~min}$ to obtain plasma samples. Each sample was analyzed as follows: A $100-\mu \mathrm{l}$ aliquot of a plasma sample was added to $400 \mu 1$ of $10 \%$ acetic acid and $100 \mu \mathrm{l}$ of internal standard solution $(0.1 \mu \mathrm{g}$ of 4 biphenylacetic acid $/ 100 \mu \mathrm{l}$ of acetonitrile $-0.1 \%$ phosphoric acid $(1: 1))$. The solution was centrifuged at $10,000 \mathrm{rpm}$ for $2 \mathrm{~min}$. The supernatant obtained was applied to a column of Bond Elut $\mathrm{C}_{18}$ (Varian, Harbor City, CA, USA), which was activated prior to use by passing through it $1 \mathrm{ml}$ of methanol and $2 \mathrm{ml}$ of water. The column was washed with $1 \mathrm{ml}$ of distilled water, and flurbiprofen and internal standard were eluted with $400 \mu \mathrm{l}$ of methanol. The eluate was dried with $\mathrm{N}_{2}$ gas at $40^{\circ} \mathrm{C}$. The residue was dissolved in $200 \mu \mathrm{l}$ of mobile phase and $20 \mu \mathrm{l}$ of this solution was injected on to the HPLC system equipped with a fluorescence detector, using excitation and emission wavelengths of 246 and $314 \mathrm{~nm}$, respectively. HPLC analysis was performed by using an Inertsil ODS-2 column (GL Science, Tokyo) with a mobile phase consisting of acetonitrile $-0.1 \%$ phosphoric acid $(1: 1)$ and a flow rate of $1.0 \mathrm{ml} / \mathrm{min}$.

\section{Gastric mucosal irritation}

On day 28, FP-T and flurbiprofen were administered to the rats which had been fasted for $18 \mathrm{hr}$. The animals were killed $6 \mathrm{hr}$ after the drug administrations; and the
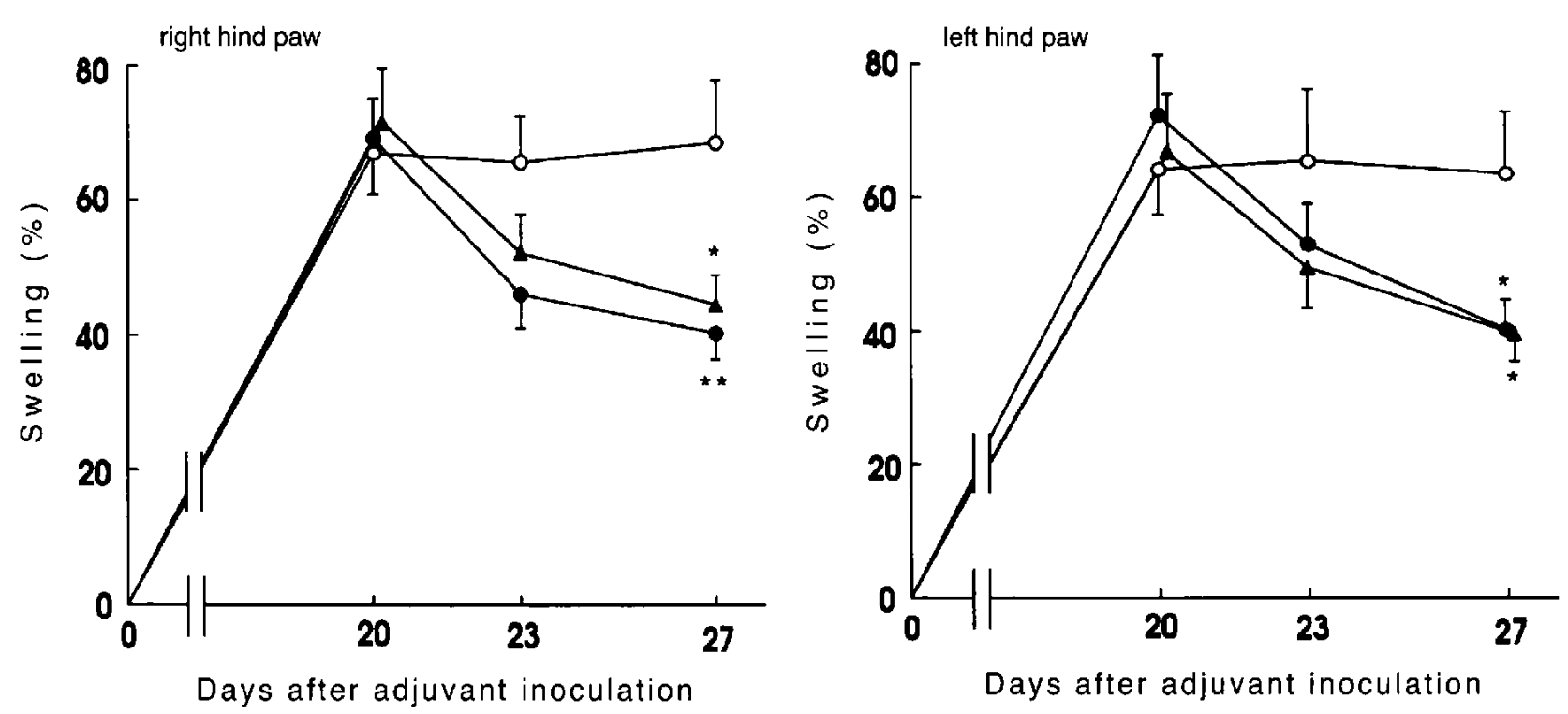

Fig. 1. Effects of FP-T and flurbiprofen (p.o.) on adjuvant-induced paw edema in rats. Treatment was started 20 days after adjuvant inoculation. FP-T was applied topically to the right hind paw for $6 \mathrm{hr}$ per day, and flurbiprofen was orally administered once a day for 8 days. The volumes of the right (left panel) and left (right panel) hind paw were measured on days 0 , 20,23 and 27. Values each represent a mean \pm S.E.M. $(\mathbf{n}=10)$. $\bigcirc$ : non-treated control; $:$ :FP-T; $\mathbf{\Delta}$ : flurbiprofen, $0.75 \mathrm{mg} / \mathrm{kg}$, p.o. ${ }^{*} \mathrm{P}<0.05,{ }^{* *} \mathrm{P}<0.01$, significantly different from the non-treated control group by one-way analysis of variance and Dunnett's Multiple Range test. 
stomach of each was excised, inflated by $10 \mathrm{ml}$ of $10 \%$ formalin solution and immersed in the same solution for $2 \mathrm{hr}$. The length of gastric irritation in the stomach was measured according to Okabe et al. (4).

\section{Statistical analyses}

Results are expressed as means \pm S.E.M. Values were statistically examined by one-way analysis of variance (ANOVA) followed by Dunnett's Multiple Range test, Student's $t$-test or the Mann Whitney $U$-test. A P value of less than 0.05 was regarded as significant.

\section{RESULTS}

\section{Effects of FP-T on adjuvant arthritis in rats}

The anti-inflammatory effect of FP-T was evaluated by inhibition of hind paw edema in rats with adjuvant arthritis. The effect of FP-T on adjuvant arthritis was a significant inhibition in both the FP-T-applied right paw and the non-applied left paw. The degree of inhibition by FP-T on swelling in the FP-T-applied and non-applied paws were the same. The anti-inflammatory effect by FP$T$ was the same as that seen with oral administration of flurbiprofen (Fig. 1). Body weight gain by treatment with topical application of FP-T and oral administration of flurbiprofen is given in Table 1. Body weight significantly increased with these treatments.

\section{Plasma concentrations of flurbiprofen}

The profiles of plasma concentration of flurbiprofen after topical application of FP-T and oral administration of flurbiprofen are shown in Fig. 2. Although the maximum plasma concentration of flurbiprofen after oral administration of flurbiprofen was higher compared to the case of topical application of FP-T, the flurbiprofen levels in the plasma were much the same as those observed with topical application of FP-T and oral administration of flurbiprofen.

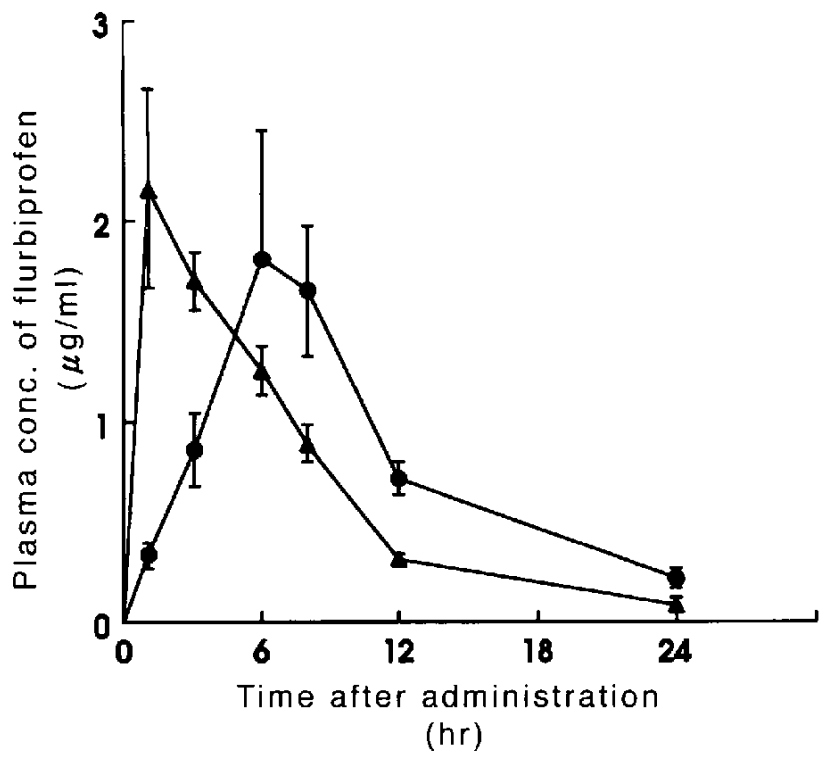

Fig. 2. Plasma concentration of flurbiprofen after topical application of FP-T and oral administration of flurbiprofen to rats. FP-T $(3.5 \mathrm{~cm} \times 4 \mathrm{~cm})$ was applied topically to the right hind paw for $6 \mathrm{hr}$, and the flurbiprofen $(0.75 \mathrm{mg} / \mathrm{kg}, \mathrm{p} . \mathrm{o}$.) was orally administered. Values each represent a mean \pm S.E.M. $(n=5)$. : FP-T, $\Delta$ : flurbiprofen (p.o.).

\section{Effect of FP-T on gastric mucosae}

The incidence of gastric damage $6 \mathrm{hr}$ after topical application of FP-T and after oral administration of flurbiprofen was $50 \%$ and $80 \%$, respectively. The ulcer index for FP-T was significantly lower compared to that for the oral administration of flurbiprofen (Table 2).

\section{DISCUSSION}

FP-T was developed as an adhesive tape with a high percutaneous absorption capacity and low gastric ulcerogenesity. We found that topical application of FP-T suppressed both FP-T applied paw edema and non-

Table 1. Body weight gain after topical application of FP-T and oral administration of flurbiprofen to adjuvant arthritic rats

\begin{tabular}{|c|c|c|c|}
\hline \multirow{3}{*}{ Groups } & \multirow{3}{*}{$\mathrm{n}$} & \multicolumn{2}{|c|}{ Body weight (g) } \\
\hline & & \multicolumn{2}{|c|}{ days after adjuvant inoculation } \\
\hline & & day 20 & day 27 \\
\hline Non-treated control & 10 & $190.1 \pm 6.7$ & $211.3 \pm 8.5$ \\
\hline FP-T $(3.5 \mathrm{~cm} \times 4 \mathrm{~cm}) 6 \mathrm{hr} /$ day & 10 & $190.7 \pm 3.4$ & $222.4 \pm 3.1^{* * *}$ \\
\hline Flurbiprofen, $0.75 \mathrm{mg} / \mathrm{kg}, \mathrm{p} .0 . /$ day & 10 & $184.6 \pm 7.1$ & $211.3 \pm 6.3^{*}$ \\
\hline
\end{tabular}

The treatment was started 20 days after adjuvant inoculation. FP-T was applied topically to the right hind paw for $6 \mathrm{hr}$ per day, and flurbiprofen was orally administered once a day for 8 days. Values each represent the mean \pm S.E.M. ${ }^{*} \mathrm{P}<0.05$, ${ }^{* * *} \mathrm{P}<0.001$, significantly different from day 20 by Student's $t$-test. 
Table 2. Gastric lesion induced by topical application of FP-T and oral administration of flurbiprofen in adjuvant arthritic rats

\begin{tabular}{lcc}
\hline Groups & Incidence of lesions & Ulcer index (mm) \\
\hline FP-T $(3.5 \mathrm{~cm} \times 4 \mathrm{~cm}) 6 \mathrm{hr} /$ day & $5 / 10(50 \%)$ & $0.95 \pm 0.34^{*}$ \\
Flurbiprofen, $0.75 \mathrm{mg} / \mathrm{kg}, \mathrm{p} .0 . /$ day & $8 / 10(80 \%)$ & $5.14 \pm 2.08$ \\
\hline
\end{tabular}

Ulcer index values each represent the mean \pm S.E.M. of 10 animals. ${ }^{*} \mathrm{P}<0.05$, significantly different from the group given flurbiprofen at $0.75 \mathrm{mg} / \mathrm{kg}, \mathrm{p} .0 . /$ day by the Mann-Whitney $U$-test.

applied paw edema of rats with adjuvant arthritis. Antiinflammatory effects of topical application of poultices or ointments containing NSAIDs are attributed to actions on inflamed tissue below the applied site, without any systemic influence, and these externally applied compounds are free from adverse effects that develop after oral administration of such drugs (2). However, we found that the anti-inflammatory effects of FP-T were exerted on not only FP-T-applied paw edema but also non-applied paw edema, with the same potency. Oral administration of flurbiprofen $(0.75 \mathrm{mg} / \mathrm{kg} /$ day $)$ also suppressed both the right and left paw edema. The activities of orally administered flurbiprofen were the same as those of topically applied FP-T. It seems that the anti-inflammatory effect of FP-T cannot be explained only by the action of flurbiprofen permeating the inflamed tissue below the applied site. The distribution of flurbiprofen absorbed transdermally may be mainly via the blood stream. The increase of body weight by FP-T or oral administration of flurbiprofen suggests that the systemic condition of rats was improved by these treatments. In addition, although the time-lag effect was observed in the case of topical application of FP-T, the plasma concentrations of flurbiprofen after topical application of FP-T were the same as those after oral administration of flurbiprofen (5). Furthermore, we preliminarily examined the effect of FP$T$ applied to the dorsal skin on hind paw edema induced by carrageenin in rats. The FP-T applied to the dorsal skin significantly suppressed the paw edema (data not shown). These results indicate that the anti-inflammatory effects of FP-T may be exerted via the systemic circulation of flurbiprofen absorbed transdermally. Further studies on FP-T, such as the dose-response relationship of the anti-inflammatory effect or analgesic effect, may be necessary.

Radermacher et al. (6) described drug concentrations in synovial fluid after cutaneous application of diclofenac gel in inflammatory and degenerative disease, at least $85 \%$ of the dose was considered to penetrate via the systemic circulation. Singh and Roberts (7) reported that local, direct penetration of NSAIDs was evident up to a depth of about 3 to $4 \mathrm{~mm}$ below the applied site, with distribution to deeper tissue being predominant through the systemic blood supply. This evidence supports our postulation.

On the other hand, gastric damages following topical application of FP-T was lower compared to findings in the case of oral administration of flurbiprofen, yet the anti-inflammatory activities were almost equivalent. Gastric damage caused by some acidic NSAIDs is due to direct actions on gastric mucosa $(8-10)$.

The transdermal delivery system (TDS) has been reviewed by Cleary (11). Ongoing studies of FP-T are being done to explore possible applications for local inflammatory diseases, arthropathies and rheumatoid arthritis.

\section{Acknowledgments}

We thank S. Fukui, Y. Yamaguchi and K. Tominaga of our laboratory for technical assistance.

\section{REFERENCES}

1 Squire I and Lees K: Topical drug delivery. Practitioner 236, 203-206 (1992)

2 Ogiso T, Ito $\mathrm{Y}$, Iwaki $\mathbf{M}$ and Atago $\mathrm{H}$ : Prediction of plasma concentration profile during single and repeated skin applications of indomethacin and its calcium salt ointments. J Pharmacobiodyn 10, 384-389 (1987)

3 Winder CV, Lembke LA and Stephens MD: Comparative bioassay of drugs in adjuvant-induced arthritis in rats: Flufenamic acid, mefenamic acid, and phenylbutazone. Arthritis Rheum 12, 472-482 (1969)

4 Okabe S, Takeuchi K, Urushidani T, Murata T, Ishibashi A and Kasuya $Y$ : Irritative activity of indomethacin, phenylbutazone or feprazone on the gastrointestinal tract in rats and dogs. Pharmacometrics 16, 241 - 247 (1978) (Abstr in English)

5 Tojo K, Chiang CC and Chien YW: Drug permeation across the skin: Effect of penetrant hydrophilicity. J Pharm Sci 76, $123-126$ (1987)

6 Radermacher J, Jentsch D, Scholl MA, Lustinetz T and Frolich JC: Diclofenac concentrations in synovial fluid and plasma after cutaneous application in inflammatory and degenerative joint disease. Br J Clin Pharmacol 31, 537-541 (1991)

7 Singh $\mathrm{P}$ and Roberts MS: Skin permeability and local tissue concentration of nonsteroidal anti-inflammatory drugs after topical application. J Pharmacol Exp Ther 268, 144-151 (1994)

8 Frey H-H and El-Sayed MA: Concentrations of acidic antiinflammatory drugs in gastric mucosa. Arch Int Pharmacodyn 
Ther 230, 300-308 (1977)

9 Imai $\mathrm{T}$, Maeda $\mathrm{T}$, Otagiri $\mathrm{M}$ and Uekama $\mathrm{K}$ : Improvement of absorption characteristics and reduction of irritation on stomach of flurbiprofen by complexation with various cyclodextrins. Xenobiol Metab Dispos 2, 657-664 (1987) (Abstr in English)

10 Imai T, Fukuhara A, Ueda I and Otagiri M: An evaluation of an anti-inflammatory-histamine $\mathrm{H}_{2}$ antagonist drug complex on gastric erosions in the rat. J Pharmacol Exp Ther 265, 328-333 (1993)

11 Cleary GW: Transdermal delivery systems: A medical rationale. In Topical Drug Bioavailability, Bioequivalence and Penetration, Edited by Shah VP and Maibach HI, pp 17-68, Plenum Press, New York (1993) 